\title{
Competence in navigation and guided transbronchial biopsy for peripheral pulmonary lesions
}

\author{
Vanina LIVI ${ }^{2}$ *, Lina ZUCCATOSTA 2, Micaela ROMAGNOLI ${ }^{3}$, Alessia PRATICÒ 4 , \\ Lucio MICHIELETTO ${ }^{5}$, Laura MANCINO ${ }^{5}$, Lorenzo CORBETTA ${ }^{6}$
}

${ }^{1}$ Pneumologia Interventistica Interaziendale, Policlinico S. Orsola Malpighi, Maggiore Hospital, Bologna, Italy; ${ }^{2}$ Operative Unit of Pneumology, Ospedali Riuniti University Hospital, Ancona, Italy; ${ }^{3}$ Operative Unit of Interventional Pneumology, Policlinico Sant'OrsolaMalpighi Hospital, Bologna, Italy; ${ }^{4}$ Operative Unit of Pneumology, Perugia University Hospital, Perugia, Italy; 5 Operative Unit of Pneumology, Ospedale dell'Angelo, Mestre, Venezia, Italy; ${ }^{6}$ Operative Unit of Interventional Pneumology, University of Florence, Florence, Italy

*Corresponding author: Vanina Livi, Pneumologia Interventistica Interaziendale, Policlinico S. Orsola Malpighi, Maggiore Hospital, Bologna, Italy. E-mail: vanina.livi@aosp.bo.it

$\mathrm{W}$ ith the recent spread of computed tomography (CT) screening, a large number of peripheral lung lesions were found. Bronchoscopy is one of the main techniques used for sampling suspicious peripheral lesions. In addition, in recent years, a large number of tumor specimens have been required to determine the best chemotherapy regimen for each patient; this personalized approach is known as precision medicine. Besides fluoroscopy, several guidedbronchoscopy technologies have recently been developed to improve the yield of transbronchial biopsy (TBB) for diagnosing peripheral lesions, such as radial endobronchial ultrasound, electromagnetic bronchoscopy navigation (EBN), virtual bronchoscopy navigation (VBN).

\section{Knowledge of the disease}

A peripheral pulmonary lesion (PPL) is defined as a lesion that is not seen within the bronchial tree at fiberoptic bronchoscopy. With the widespread use of CT, peripheral pulmonary lesions have become common incidental findings 
in routine medical practice. It is fundamental to be able to distinguish lung cancer from benign lesion and transthoracic needle aspiration (TTNA) or bronchoscopy represent less invasive diagnostic methods than surgical biopsy. ${ }^{1}$

TTNA under CT-guidance has a sensitivity for malignancy between $74 \%$ and $96 \%$ (it depends on the size of the lesions, distance of the pleura and biopsy technique employed) and a specificity of nearly $100 \% .^{2-4}$ However, this procedure has a high incidence of complications; a meta-analysis of 15,865 patients showed a pneumothorax rate of $15 \%$ with $>6 \%$ of all procedures requiring the insertion of a chest tube. The risk of pneumothorax is related to the number of biopsies, distance to the pleura, size of the lesion and degree of lung emphysema. The incidence of hemoptysis after TTNA was $1-5 \% .^{5}$

The American College of Chest Psysician (ACCP) Guidelines reported that with bronchoscopy the sensivities for PPLs larger and smaller than $2 \mathrm{~cm}$ were $63 \%$ and $34 \%$ respectively. The advantage of the bronchoscopic diagnosis of PPLs over CT-guided TTNA is the lower complication rate (PNX rate of $<3 \%$ ). Conventional bronchoscopy of PPLs is usually performed under X-ray fluoroscopy and the main sampling procedures are transbronchial lung biopsy (TBLB), transbronchial needle aspiration (TBNA), brushing and cryobiopsy. Rivera et al. performed a metaanalysis that showed that TBNA alone or combined with TBB is superior to TBB with forceps alone in diagnosing PPLs. ${ }^{2}$ Compared with central lesion, it is difficult to guide a bronchoscope and sampling instruments to PPLs. The bronchoscopist normally estimates the 3 -dimensional distribution of the bronchus by referring to 2-dimensional planar axial slices on pre-examination CT, but this method is inaccurate for bronchi that are more peripheral than the subsegmental bronchi. ${ }^{6}$ The diagnostic yield depends on the size of the lesion, the presence of "bronchus sign," the visibility under fluoroscopy when used and the skill of the operator.

\section{Knowledge of instruments}

Fluoroscopy guidance

Fluoroscopy should be employed with TBB and TBNA in cases of localized or focal parenchymal lung disease. The use of fluoroscopy during TBB has been shown to increase the diagnostic yield in focal lesions. The number of biopsy specimens required for optimal diagnostic yield has been reported as being between 4 and 10. The 2013 BTS Guidelines recommend that at least five samples should be obtained in cases where endobronchial tumor is visible and that at least five or six samples should be obtained in cases of interstitial lung disease.

\section{Radial EBUS}

EBUS miniprobe is a procedure used to collect samples of localized peripheral lesions. It is used in conjunction with the reconstruction provided by $\mathrm{CT}$ scans and assisted by fluoroscopy or virtual bronchoscopy navigation (VBN) during the procedure (Figure 1). It is therefore an improved way of performing a TBLB of a peripheral lesion. ${ }^{7,8}$ Many bronchoscopy units perform procedures for such lesions only using a bronchial washing or X-ray fluoroscopy guided biopsy, with a diagnostic yield of about $40 \%$.

Comparative studies have shown the improved diagnostic yield of adding EBUS miniprobe, compared with fluoroscopy alone. ${ }^{9}, 10$ The miniprobe can be used to sample lesions in distal bronchi, bronchioles and alveoli. According to the sonographic features of the target lesion, the relationship between the lung lesion and the position of the probe is classified into three patterns: within, adjacent and out-

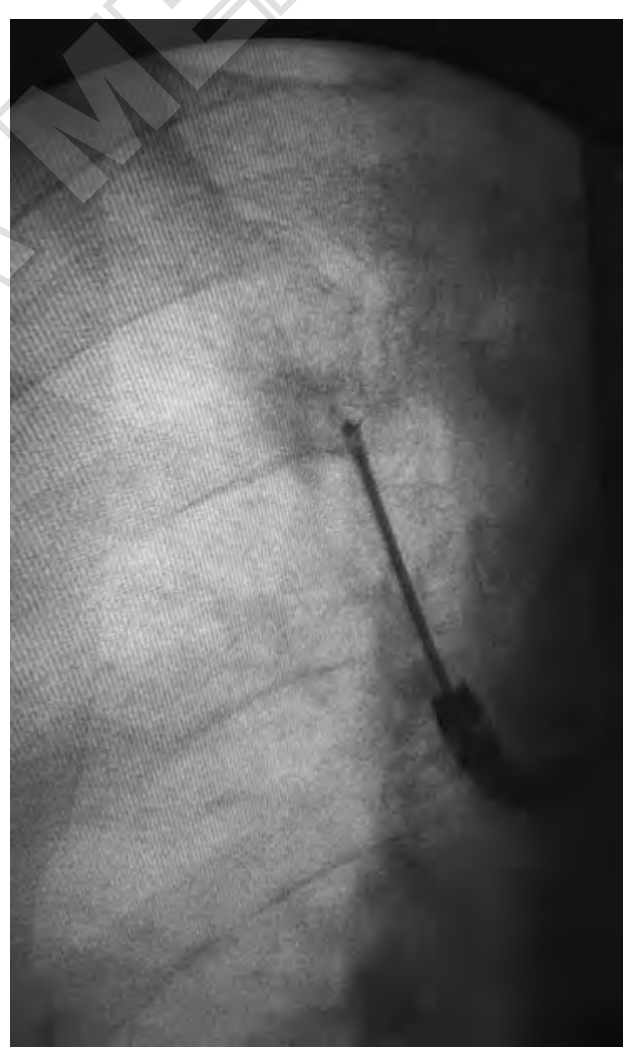

Figure 1.- Reconstruction provided by CT scan and assisted by fluoroscopy or virtual bronchoscopy navigation during the procedure. 


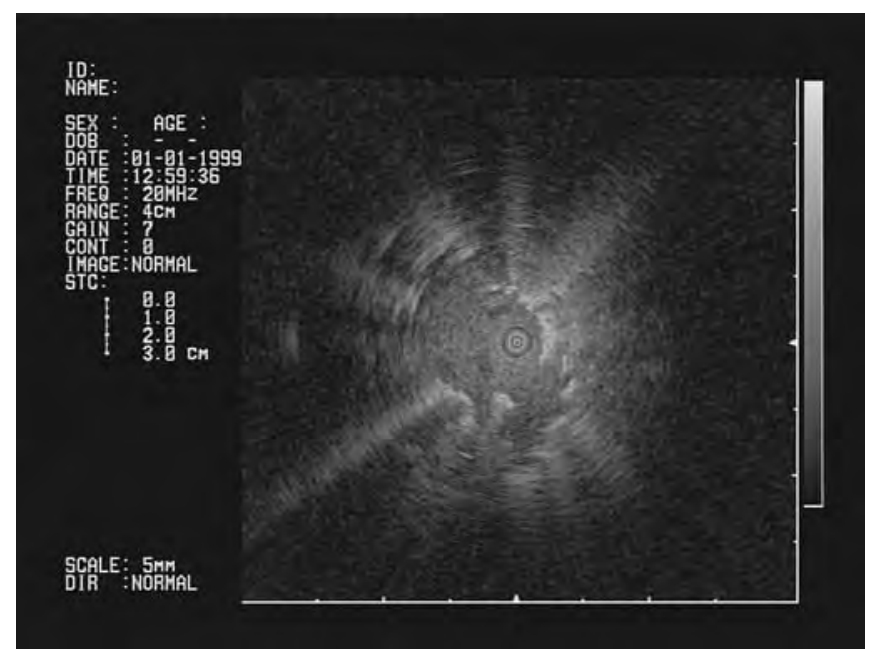

Figure 2.-Compact thickening marginal radial probe.

side the lesion (Figure 2, 3, 4). Bronchus sign is associated with the diagnostic sensitivity of miniprobe. On the basis of the relationship between the target lesion and the nearest bronchi are defined three types of Tc bronchus sign: type A when the TC images show the bronchus clearly extending inside the target lesion, type $\mathrm{C}$ when no bronchus can be detected within the lesion, and type B when the CT finding cannot be categorized as either type A or C. ${ }^{11}$ The sensitivity for primary malignancy is $>70 \%$. Some regions of the lung are less easy to access with any form of transbronchial lung biopsy, and this is true for miniprobe EBUS in the left upper lobe. A wide range of localized infectious and inflammatory lesions can also be sampled using miniprobe EBUS. The techniques for miniprobe EBUS are the same as those currently used for sampling peripheral lesions with fluoroscopy.

There are two additional aspects to training practitioners for this technique: first, the use of the plastic guide sheath through which the miniprobe is passed; second, when the probe enters a lesion, an ultrasound image is displayed in real time. During the procedure, the image is used primarily to confirm that the lesion has been localized, and interpretation of the image is used simply to show that there is a circumferential ultrasound shadow corresponding to a lesion, as opposed to uninvolved lung surrounding the miniprobe (Figure 2, 3, 4). ${ }^{12-15}$ An important aspect of the miniprobe technique with the use of the guide sheath is the very low complications rate, compared with standard transbronchial lung biopsy. With this technique, bleeding and pneumothorax are around $\pm 1 \%$, whereas they are about five times higher $( \pm 5 \%)$ in

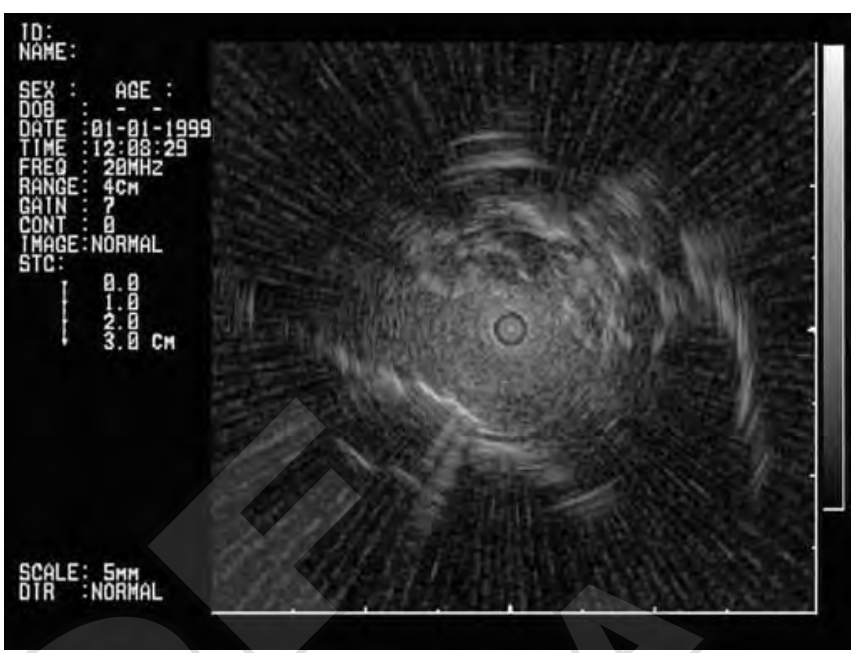

Figure 3.-Compact thickening with colliquated areas inside.

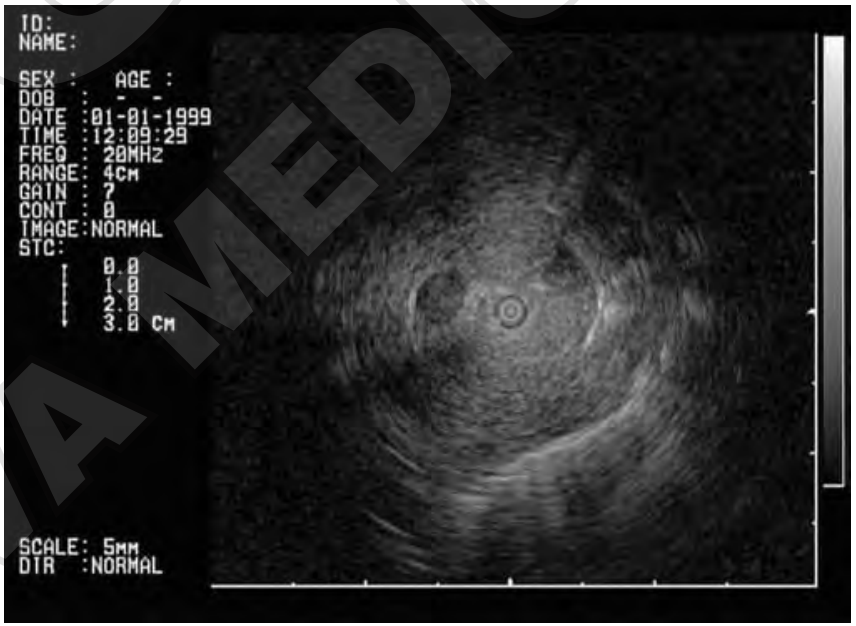

Figure 4.-Compact thickening radial central probe.

standard transbronchial lung biopsy, including reports of major bleeding. 13,14

EBUS miniprobe with or without guide sheat is indicated for diagnosis of peripheral lesions and ATS guidelines recommends radial EBUS as adjunctive imaging modality in patients with suspected lung cancer in whom a tissue diagnosis is required. ${ }^{16}$ It is indicated also in the diagnosis of benign peripheral lesions and in cavitary lung lesions. The EBUS miniprobe technique can also be used for obtaining specimen for molecular analysis of EGFR. The diagnostic yield of radial miniprobe is $60-70 \%$ when it used alone and increased until $84 \%$ with fluoroscopic guide and when was used in combination with virtual bronchoscopic navigation the overall diagnostic yield increased to $63.3-84 \% .{ }^{11}, 17$ 
VBN

VBN is a method that produces 3-dimensional, virtual bronchoscopy images of the tracheal and bronchial lumens. These images, that can be used as a guide to navigate bronchoscope towards peripheral pulmonary lesions, are prepared from a continuous volume data of a helical CT. ${ }^{18} \mathrm{VBN}$ can be performed using standard software for virtual bronchoscopy or specific systems which are commercially available (DirectPath system, Cybernet System Inc; Lung Point, Broncus Medical Inc). The key problem of the general-purpose software for virtual bronchoscopy is that the accuracy of the images depends critically on the threshold used to differentiate between the airway wall and the airway lumen. Inappropriate thresholds may lead to overlook bronchial bifurcations in the peripheral airways, which in turn may lead to unreliable navigation towards the target. ${ }^{19}$ Furthermore, when the scope is rotated into the airways, the true bronchial image does not correspond anymore to the virtual bronchoscopy image prepared earlier. As a consequence, the virtual bronchoscopy image should be rotated to adapt it to the real broncoscopy image at each bifurcation in order to maintain a reliable realtime navigation. ${ }^{20}$ Specific systems for VBN are meant to overcome these shortcomings. In fact, they automatically search for a bronchial route by simply setting the target lesion, and automatically match the virtual images with the actual images for reliable bronchoscopy navigation to the target lesion (Figure 5, 6). ${ }^{21}$

EBN

EBN is a procedure that utilizes electromagnetic technology to localize lesions in the lung and to guide sampling instruments through the airways towards the lesion. ${ }^{22}$ The two currently available EBN systems are the superDimension navigation system (Medtronic, Minneapolis, MN, USA) and the SpiN system (Veran, Veran Medical Technologies SPiNDrive System, St Louis, MO, USA).

The superDimension ${ }^{\mathrm{TM}}$ navigation system, which is the most commonly used EBN system by far, is based of four components. First, a hardware component which includes the computer, monitors, and, above all, an electromagnetic localization board which generates a low-frequency electromagnetic field surrounding the thorax of the patient. Due to the possible influence of metallic objects the field has to be calibrated for every endoscopic suite. Second, a licensed software that converts previously acquired CT images into multiplanar virtual bronchoscopy of the patient's airways. Third, a steerable locatable guide (1 mm in diam-

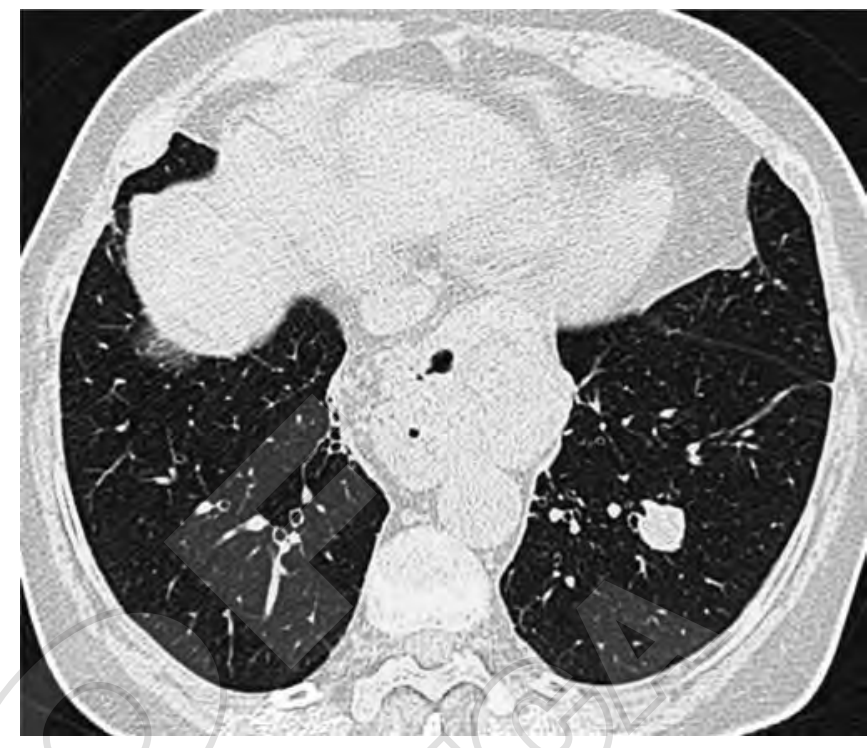

Figure 5. $-\mathrm{CT}$ scan from a 64 -years-old woman with a $13 \mathrm{~mm}$ pulmonary nodule in the left lower lobe suspicious for lung cancer.
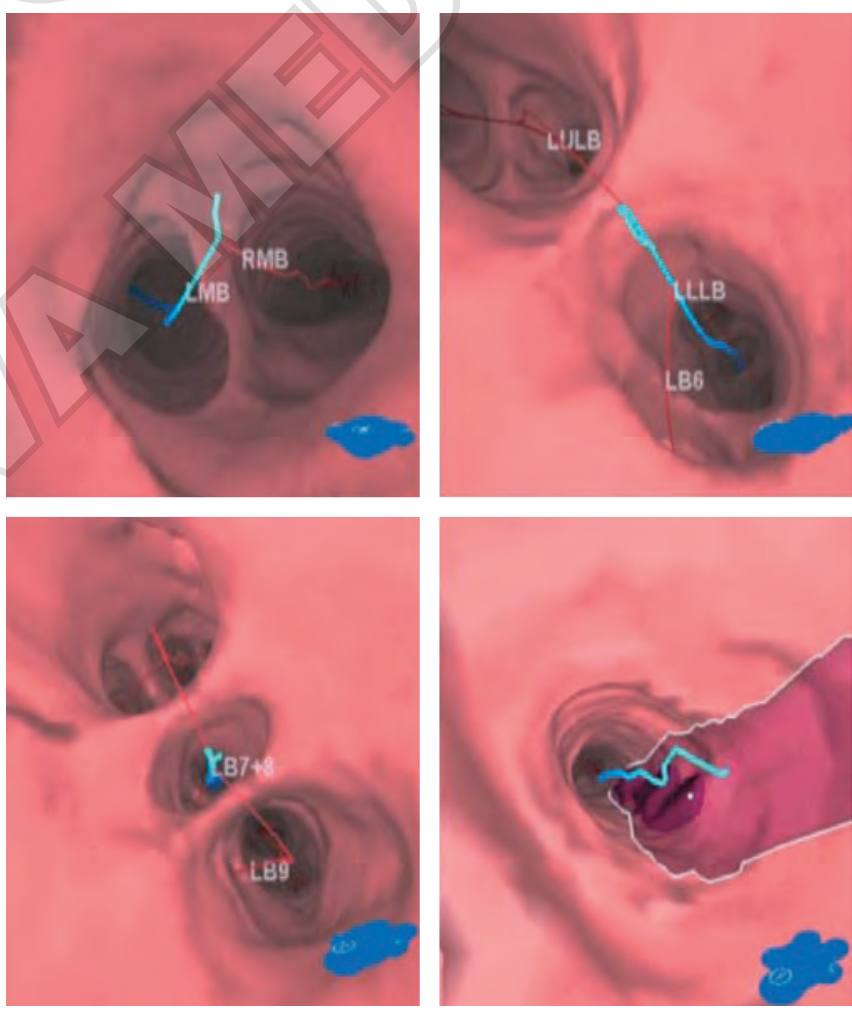

Figure 6.-Virtual bronchoscopy with the Lung Point System (Broncus Medical, Mountain View, CA, USA) for a suspicious nodule in the left lower lobe (CT scan Figure 5). The pathway to the lesion is overlaid as a blue line. RMB: right main bronchus; LMB: left main bronchus; LULB: left upper lobe bronchus; LLLB: left lower lobe bronchus; LB: left bronchus. 
eter and $8 \mathrm{~mm}$ in length), which can navigate in the bronchial tree and is connected to a flexible metal cable. Fourth, a dedicated guide sheath, which can be used as an extended working channel (EWC, $130 \mathrm{~mm}$ in length and $1.9 \mathrm{~mm}$ in diameter) after the locatable guided is removed from it.23,24

The SpiN system uses specific stickers (vPads ${ }^{\mathrm{TM}}$ ) equipped with electromagnetic sensors which are placed on the patient's chest and remain in place during the procedure to help guide navigation and track patient breathing. The system also includes an electromagnetic field generator and a licensed, laptop-based software that converts the scans and creates a dynamic 3D map leading to the target lesion. Interestingly, the $\mathrm{vPads}{ }^{\mathrm{TM}}$ enable automatic registration and dynamic referencing throughout the procedure, and communicate with respiratory gating technology to track and update respiratory movements.

Several individual studies, as well as two meta-analysis, assessed a key aspect of the procedure, namely the factors possibly influencing the diagnostic yield. Six statistically significant variables were identified by univariate by individual studies. Lower lobe location of the PPL was associated with decreased yield, whereas greater nodule size, presence of a bronchus sign, lower registration error (AFTRE: Average Fiducial Target Registration Error), nodule visualization with a radial EBUS probe, and catheter suction technique increase diagnostic yield. A multivariate analysis was performed in a single study, and a single factor (presence of a bronchus sign, OR 7.6, 95\% CI 1.8-31.7) was found to predict independently a positive diagnosis. ${ }^{25}$ One metanalysis analyzed the study level characteristics associated with significant modification of EBN performance and concluded that general anesthesia and rapid on-site evaluation are associated with statistically better diagnostic yield.

The success rate is a key aspect of a diagnostic procedure. Individual studies, metanalysis and registries did evaluate the performance characteristics of EBN for the diagnosis of PPLs. The diagnostic yield of EBN ranged from $49 \% 26$ to $87 \% 27$ in individual studies, with a pooled diagnostic yield of $65 \%$ in the most accurate metanalysis published to date. ${ }^{28} \mathrm{~A}$ few key comments, however, are warranted to put these data into proper context. First, EBN is seldom used as a stand-alone procedure for the bronchoscopic approach to PPLs. In most studies, in fact, EBN was used with fluoroscopy, radial EBUS or both to confirm correct nodule sampling. Therefore, the data regarding diagnostic yield of EBN emerging from clinical trials have be taken with great caution as they may have been increased by the association with other guid- ance systems. Second, EBN has been compared only once with alternative systems used to guide bronchoscopy for the diagnosis of PPLs. Eberhardt et al., in particular, performed a randomized trial aimed at comparing the yield of EBN alone, r-EBUS alone, and both for the diagnosis of EBN. ${ }^{27}$ The diagnostic yield was 59\% for EBN, 69\% for r-EBUS, and $87.5 \%$ for the combined arm. The combination of both procedures was statistically superior to either EBN and r-EBUS as stand-alone procedures. Third, the data from a large US registry tend to suggest that when the modern guidance systems (EBN and r-EBUS) are used outside the research setting they do not perform as well as in clinical trials. ${ }^{29}$ In 2016 , Ost et al. reported the results of the AQUIRE Registry regarding the diagnostic yield of bronchoscopy for PPLs and showed that the yield of EBN alone and r-EBUS alone are $38.5 \%$ and $57 \%$, respectively. The diagnostic yield did not differ significantly based on the size of the lesion and it is similar between the two commercially available systems. In conclusion, the yield of EBN seems at most similar to that of other guided endoscopic procedures and compared with the other guidance systems it is significantly costlier, more time consuming (30-40 min including preprocedural plannig and simulation), and requires a lot of resources. ${ }^{28}$

Adverse events following EBN procedures are uncommon. Pneumothorax is the most frequently reported complication, with pooled rates around 3\% and much lower than the $20 \%$ rate reported for transthoracic needle biopsy and EBN-guided TTNA. 28,30

In the Clinical Evaluation of super Dimension Navigation System for Electromagnetic Navigation Bronchoscopy study, the largest prospective, multicenter EBN study conducted to date, in 1000 patients, the EBN-related PNX rates were $4.9 \%$ overall and $3.2 \%$ for PNX requiring chest tube or hospitalization. ${ }^{31}$

\section{Other potential applications}

A new method using the VBN system, namely bronchoscopic transparenchymal nodule access (BTPNA), differs from the other guidance systems. Using an exclusive software (Archimedes, Broncus Medical Inc), a route is planned for approaching the target lesion throuought the lung parenchyma avoiding blood vessels, and its point-ofentry $(\mathrm{POE})$ is set in the central airway.

The POE is identified under virtual guidance and a coring needle is used to penetrate the airway wall. The hole at the POE is then enlarged by a balloon dilator. Later, a direct tunnel with a sheath reaching the lesion is prepared along the planned route in the lung parenchyma by 
matching the VBN system and real fluoroscopic images for sample collection. This method may be promising with lesions without bronchus sign or not approachable bronchoscopically. However, investigations of BTPNA safety is necessary. ${ }^{32,33}$

EBN can also be used for placing fiducial markers for robotic stereotactic radiosurgery for a precise tumor ablation and to preserve healthy tissue 34,35 and to mark PPLs to identify the location of the lesion during thoracoscopic surgery. ${ }^{36,37}$

Endoluminal brachytherapy under combined EBUS/ EBN guidance may be suitable for selected patients to spare major damage to radiosensitive surrounding structures, but it has high costs and it has a complicated logistics. ${ }^{38}$

Initial publications on bronchoscopic radiofrequencyinduced tissue ablation (RFA) are available but its role is not sufficiently clear.

The possibility of applying curative bronchoscopic treatments to small pulmonary nodules in nonsurgical patients may be a option for patients with limited surgical fitness or previous radical treatment but even though the technologies are attractive and data from pilot trials are encouraging, more studies are needed to establish selection criteria and real utility.

In the future, the goal should be to diagnose and to treat early stages of lung cancer endoscopically in one procedure. 39,40

\section{Core basic skills}

Prior Experience Requirements: Trainees must have completed training as described in articles on "Flexible bronchoscopy and basic biopsy technique" and "EBUSTBNA." Furthermore $>10$ standard transbronchial lung biopsies, having achieved competency in this technique, including familiarity with fluoroscopy X-ray.

\section{Radial EBUS}

Core basic skills in the use of EBUS miniprobe are:

- ability to localize the lesions in relation to the segment involved in CT scan;

- ability to guide the probe to the involved segment and lesion;

- ability to interpret the position of the probe in relation to the peripheral lesion;

- ability to distinguish anatomic structures and pathologic lesions in endosonography;

- use of the plastic guide sheath through which the miniprobe is passed;
- management of specimens for cytology, histology and molecular tests;

- prevention and management of possible complications (bleeding, pneumothorax, infections). ${ }^{41,42}$

\section{EBN/VPN}

The basik skills to perform endobronchial navigation procedures are:

- competency in flexible bronchoscopy and ancillary sampling methods (endobronchial biopsy, TBB with forceps or cryo-biopsy, TBNA);

- correct interpretation of chest CT scan and ability to localize the lesions in relation to the segment involved in CT scan;

- ability to use the VBN/EBN software for the planning of procedures;

- ability to perform the procedure, to reach the lesion and to achieve the sampling;

- management of specimens for cytology, histology and, if requested, for molecular tests;

- prevention and management of possible complications (pneumothorax, bleeding, complications related to local anesthesia or sedation).

\section{Procedural description and sedation}

\section{EBN/VPN}

Both VBN and EBN consist of two key phases, namely planning and procedure (or guidance). In the planning phase, a virtual bronchoscopic image and a pathway to a target lesion are obtained with specific planning software modules starting from CT scans acquired with specific settings (slice thickness and intervals). In the second phase, bronchoscopy with biopsy of the target lesion is carried out.

1. SuperDimension Planning software:

a) upload the patient study in the appropriate DICOM format;

b) mark registration points;

c) review 3D map;

d) mark target;

e) plan pathway + add a new pathway;

f) mark the affected area, towards which navigation is targeted. Registration points are marked at the main carina, right upper lobe carina, right middle lobe, right lower lobe, left upper lobe and left lower lobe: these are used to match the patient to the scan. More recent software allows the system to automatically perform the registration and match the patient to the scan. A route to the area can either be planned manually or suggested by the software; 


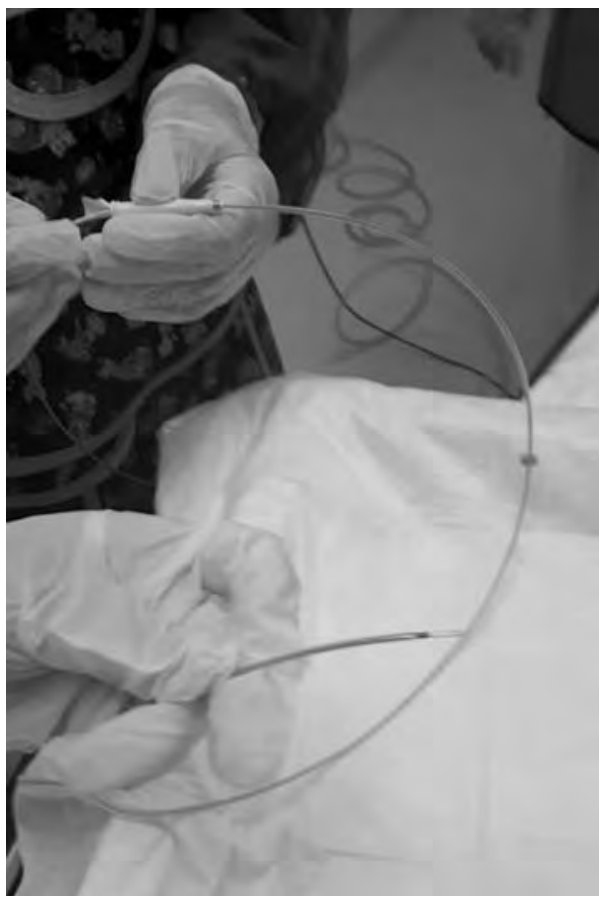

Figure 7.--Procedural steps.

g) export the plan via USB.

2. Performing the EBN procedure:

a) preparing system and patient (location board, three location pads and set-up of the patient);

b) preparing the navigation catheter and the Locatable Guide (LG);

c) navigation and biopsy. In the final navigation phase, a steerable catheter is used to navigate to the lesion. The system displays a multiscreen view. The navigation is performed using a specially designed LG. It can be rotated 360 degrees using the small dial on the neck of the catheter. Then, by pulling the neck back, it is flexed in the direction indicated by the arrow on the catheter. The setting of the directional steerable guide inside the catheter is shown in the lower right portion of the navigation screen by arrows seen in the orange circle. Progress is followed by the relative locations of the purple dot. Thus, the LG is steered through the tracheobronchial tree until the target is reached. Once the LG tip is aligned with and in close proximity to the target lesion, the EWC is locked onto the bronchoscope, the LG is removed, leaving in place an EWC through which a biopsy forceps or other tools can be inserted and used.

d) Preparing for a biopsy: once the LG tip is aligned with and in close proximity to the target lesion the EWC is locked onto the bronchoscope, the LG is removed, leaving in place an EWC through which a biopsy forceps or other tools can be inserted and used.

Sedation EBN is carried out in the vast majority of cases under moderate (intravenous opioids + benzodiazepines) or deep sedation, as constant motion, rapid breathing, and coughing can compromise adequate navigation and/or sampling, while the duration of EBN, significantly longer that of conventional bronchoscopy, would require a very high patient's tolerance. ${ }^{43}$ In the largest study published to date, approximately $80 \%$ of the patients underwent the procedure under general anesthesia. ${ }^{31}$ No head-to-head comparison of safety and yield of EBN performed under moderate or deep sedation is available in the literature. Only two studies tried to evaluate the influence of general anesthesia on the procedure, but the statistical power was not enough to allow for definitive conclusions to be drawn. ${ }^{27,44}$ One meta-analysis, however, verified that studies using EBN under general anesthesia showed better diagnostic yields $(69.2 \%$ versus $57.5 \%, \mathrm{P}=0.02){ }^{28}$

\section{Radial EBUS}

Procedural steps (Figure 7, 8, 9, 10):

1) preparation of miniprobe, biopsy forceps and brush in all cases; 


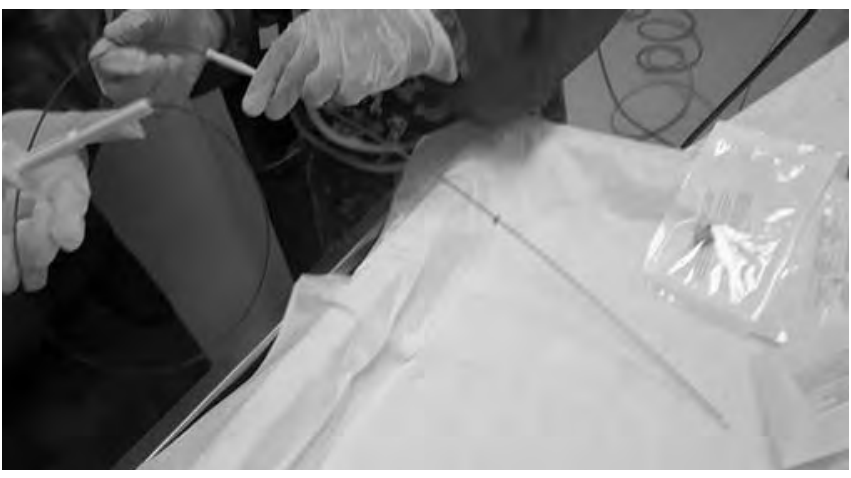

Figure 10.- Insertion of forceps into the guide-sheath.

2) print out bronchoscopic picture of the relevant lobe, labelling all segmental branches up to fifth order where possible and labelling the branch where the probe was passed to give entry to the lesion: data to be recorded in the Logbook;

3 ) enter the lesion to obtain an ultrasound image (either central or peripheral); possible in $75 \%$ of cases of lesions $>2 \mathrm{~cm}$;

4) take pictures of the EBUS image and attribute an EBUS score (I/II/III) before obtaining the histological report;

5) fit the plastic sheath to the miniprobe before the procedure; once the probe has entered the lesion, the probe is removed, while the sheath is left in place;

6) biopsy forceps and TBNA are passed through this sheath to collect the samples. In fact, this makes the whole process of doing the TBB easier because there is no need to re-localize the lesion each time;

7) interpretation and reporting of test results.

\section{Theoretical and pratical training}

\section{Radial EBUS}

- Radiology training with interpretation of chest CT scans and relationship between airways and lesions (Xrays);

- theoretical lessons and practice with EMN and VPN systems and software;

- attending courses on the method in Specialized High Volume Centers;

- observation of procedures in order to gain theoretical competence;

- performance of the procedure on plastic (Ultrasonic Bronchoscopy Simulator) and animal models (at least 30 procedures).

\section{EBN/VPN}

EBN has been recommended in the guidelines of the ACCP, with a $1 \mathrm{C}$ recommendation grade, in patients with PPLs difficult to reach with conventional bronchoscopy, if the equipement and expertise are available 2,45 and was also recently added to the interventional pulmonology fellowship accreditation standards. ${ }^{46}$

- Theoretical lessons and practice on imaging of pulmonary nodules and the mediastinum;

- practical session and simulation with planning software;

- basic training on the use of the navigation system.

- simulation training for use of EMN and VPN with manikin or animal/cadaver lungs: artificial target could be prepared with injectable substances (injected either bronchoscopically or transthoracically), visible by chest CT, echogenic EBUS radial probe, aqueous solution of $10 \%$ (by weight) bovine skin gelatine (Sigma-Aldrich, St Louis, MO), 2\% agar (Sigma-Aldrich), and $0.1 \%$ iodinated contrast (Optiray 350, Malinckrodt, St Louis, MO, USA), heated to $90^{\circ} \mathrm{C}$ during mixing and maintained as a solution at $45^{\circ} \mathrm{C}$ to $50^{\circ} \mathrm{C}$ before injection. Approximately 5 to $7.5 \mathrm{~mL}$ of this solution is used for each artificial tumor target.

\section{Quantitative and qualitative assessment}

\section{Radial EBUS}

\section{Quantitative measures:}

A minimum of 20 procedures per year are probably necessary to achieve and mantain minimum competency.

Qualitative measures:

- correlation between the image and the final histology in $>75 \%$ of cases (data to be recorded in the Logbook);

- sensitivity for malignancy: $60-70 \%$;

- typical procedure time: $30-40$ minutes;

- safety: $<1 \%$ bleeding, pneumothorax, infections. ${ }^{41,42}$

\section{EBN/VPN}

Quantitative measures:

The extent of the learning curve for $\mathrm{EBN}$ is not known. In a study of EBN-VM system, diagnostic yield was $80 \%$ in the first 25 patients enrolled compared with $87 \%$ in the last 23 subjects enrolled. ${ }^{47}$ Similar results were reported using the EBN-SD system, with diagnostic yields of $80 \%$ vs. $87.5 \%$ in the first 30 and last 30 subjects. ${ }^{48}$ Long et al. feel that new operators would benefit from both hands-on training and procedural simulation. ${ }^{49}$ 
A minimum of 20 procedures per year are probably necessary to achieve and maintain minimum competency.

Qualitative measures:

For qualitative assessment, MCQ, core-based questionnaires and creation of a specific STAT (like EBUS STAT, for instance) would be useful

Outcome measures: Correlation between the image and the final histology in $>75 \%$ of cases: to be recorded in the Logbook:

- sensitivity for malignancy: $60-70 \%$;

- typical procedure time: $30-40$ minutes;

- safety: $<1 \%$ bleeding, pneumothorax, infections. ${ }^{50}$

\section{References}

1. Gould MK, Fletcher J, Iannettoni MD, Lynch WR, Midthun DE, Naidich DP, et al.; American College of Chest Physicians. Evaluation of patients with pulmonary nodules: when is it lung cancer?: ACCP evidence-based clinical practice guidelines (2nd edition). Chest 2007;132(Suppl):108S $30 \mathrm{~S}$.

2. Rivera MP, Mehta AC, Wahidi MM. Establishing the diagnosis of lung cancer: Diagnosis and management of lung cancer, 3rd ed: American College of Chest Physicians evidence-based clinical practice guidelines. Chest 2013;143(Suppl):e142S-65S.

3. Gould MK, Donington J, Lynch WR, Mazzone PJ, Midthun DE, Naidich DP, et al. Evaluation of individuals with pulmonary nodules: when is it lung cancer? Diagnosis and management of lung cancer, 3rd ed: American College of Chest Physicians evidence-based clinical practice guidelines. Chest 2013;143(Suppl):e93S-120S.

4. Priola AM, Priola SM, Cataldi A, Errico L, Di Franco M, Campisi $\mathrm{P}$, et al. Accuracy of CT-guided transthoracic needle biopsy of lung lesions: factors affecting diagnostic yield. Radiol Med (Torino) 2007; 112:1142-59.

5. Wiener RS, Schwartz LM, Woloshin S, Welch HG. Populationbased risk for complications after transthoracic needle lung biopsy of a pulmonary nodule: an analysis of discharge records. Ann Intern Med 2011;155:137-44.

6. Dolina MY, Cornish DC, Merritt SA, Rai L, Mahraj R, Higgins WE, et al. Interbronchoscopist variability in endobronchial path selection: a simulation study. Chest 2008;133:897-905.

7. Du Rand IA, Barber PV, Goldring J, Lewis RA, Mandal S, Munavvar $\mathrm{M}$, et al.; British Thoracic Society Interventional Bronchoscopy Guideline Group. British Thoracic Society guideline for advanced diagnostic and therapeutic flexible bronchoscopy in adults. Thorax 2011;66(Suppl 3):iii1-21.

8. Gould MK, Donington J, Lynch WR, Mazzone PJ, Midthun DE, Naidich DP, et al. Evaluation of individuals with pulmonary nodules: when is it lung cancer? Diagnosis and management of lung cancer, 3rd ed: American College of Chest Physicians evidence-based clinical practice guidelines. Chest 2013;143(Suppl):e93S-120S.

9. Ernst A, Silvestri GA, Johnstone D; American College of Chest Physicians. Interventional pulmonary procedures: Guidelines from the American College of Chest Physicians. Chest 2003;123:1693-717.

10. Bolliger $\mathrm{CT}$, Mathur $\mathrm{PN}$, Beamis JF, Becker HD, Cavaliere $\mathrm{S}$, Colt H, et al.; European Respiratory Society/American Thoracic Society. ERS/ATS statement on interventional pulmonology. Eur Respir J 2002;19:356-73.

11. Minezawa $T$, Okamura $T$, Yatsuya $H$, Yamamoto $N$, Morikawa S, Yamaguchi $\mathrm{T}$, et al. Bronchus sign on thin-section computed tomography is a powerful predictive factor for successful transbronchial biopsy using endobronchial ultrasound with a guide sheath for small peripheral lung lesions: a retrospective observational study. BMC Med Imaging 2015;15:21. 12. Leong S, Shaipanich T, Lam S, Yasufuku K. Diagnostic bronchoscopy - current and future perspectives. J Thorac Dis 2013;5(Suppl 5):S498 510 .

13. Herth FJ, Ernst A, Becker HD. Endobronchial ultrasound-guided transbronchial lung biopsy in solitary pulmonary nodules and peripheral lesions. Eur Respir J 2002;20:972-4.

14. Kurimoto N, Miyazawa T, Okimasa S, Maeda A, Oiwa H, Miyazu $\mathrm{Y}$, et al. Endobronchial ultrasonography using a guide sheath increases the ability to diagnose peripheral pulmonary lesions endoscopically. Chest 2004;126:959-65.

15. Kikuchi E, Yamazaki K, Sukoh N, Kikuchi J, Asahina H, Imura M, et al. Endobronchial ultrasonography with guide-sheath for peripheral pulmonary lesions. Eur Respir J 2004;24:533-7.

16. Rivera MP, Mehta AC, Wahidi MM. Establishing the diagnosis of lung cancer: Diagnosis and management of lung cancer, 3rd ed: American College of Chest Physicians evidence-based clinical practice guidelines. Chest 2013;143(Suppl):e142S-65S.

17. Zang L, Wu H, Wang G. Endobronchial ultrasonograpy using a guide sheat tecnique for diagnosis of perpipheral pulmonary lesions. Eusjournal 2017;5:292-9.

18. Vining DJ, Liu K, Choplin RH, Haponik EF. Virtual bronchoscopy. Relationships of virtual reality endobronchial simulations to actual bronchoscopic findings. Chest 1996;109:549-53.

19. Asano F, Matsuno Y, Tsuzuku A, Anzai M, Shinagawa N, Yamazaki $\mathrm{K}$, et al. Diagnosis of peripheral pulmonary lesions using a bronchoscope insertion guidance system combined with endobronchial ultrasonography with a guide sheath. Lung Cancer 2008;60:366-73.

20. Asano F, Matsuno Y, Shinagawa N, Yamazaki K, Suzuki T, Ishida T, et al. A virtual bronchoscopic navigation system for pulmonary peripheral lesions. Chest 2006;130:559-66.

21. Asano F, Eberhardt R, Herth FJ. Virtual bronchoscopic navigation for peripheral pulmonary lesions. Respiration 2014;88:430-40.

22. Becker HD, Herth F, Ernst A, Schwarz Y. Bronchoscopic biopsy of peripheral lung lesions under electromagnetic guidance: a pilot study. J Bronchology Interv Pulmonol 2005;12:9.

23. Ozgul G, Cetinkaya E, Ozgul MA, Abul Y, Gencoglu A, Kamiloglu $\mathrm{E}$, et al. Efficacy and safety of electromagnetic navigation bronchoscopy with or without radial endobronchial ultrasound for peripheral lung lesions. Endosc Ultrasound 2016;5:189-95.

24. Eberhardt R, van der Host J. Navigational bronchoscopy in solitary pulmonary nodules. In Herth FJF, Shah PL, Gompelmann D, eds. Interventional Pulmonology [ERS monograph]. Sheffield, ERS; 2017. p. $162-175$.

25. Seijo LM, de Torres JP, Lozano MD, Bastarrika G, Alcaide AB, Lacunza MM, et al. Diagnostic yield of electromagnetic navigation bronchoscopy is highly dependent on the presence of a Bronchus sign on CT imaging: results from a prospective study. Chest 2010;138:1316-21.

26. Mahajan AK, Patel S, Hogarth DK, Wightman R. Electromagnetic navigational bronchoscopy: an effective and safe approach to diagnose peripheral lung lesions unreachable by conventional bronchoscopy in high-risk patients. J Bronchology Interv Pulmonol 2011;18:133-7.

27. Eberhardt R, Anantham D, Ernst A, Feller-Kopman D, Herth F. Multimodality bronchoscopic diagnosis of peripheral lung lesions: a randomized controlled trial. Am J Respir Crit Care Med 2007;176:36-41.

28. Gex G, Pralong JA, Combescure C, Seijo L, Rochat T, Soccal PM. Diagnostic yield and safety of electromagnetic navigation bronchoscopy for lung nodules: a systematic review and meta-analysis. Respiration 2014;87:165-76.

29. Ost DE. Reply: quantifying the benefits of peripheral transbronchial needle aspiration. Am J Respir Crit Care Med 2016;194:122-3. 
30. DiBardino DM, Yarmus LB, Semaan RW. Transthoracic needle biopsy of the lung. J Thorac Dis 2015;7(Suppl 4):S304-16.

31. Khandhar SJ, Bowling MR, Flandes J, Gildea TR, Hood KL, Krimsky WS, et al.; NAVIGATE Study Investigators. Electromagnetic navigation bronchoscopy to access lung lesions in 1,000 subjects: first results of the prospective, multicenter NAVIGATE study. BMC Pulm Med 2017;17:59.

32. Herth FJ, Eberhardt R, Sterman D, Silvestri GA, Hoffmann H, Shah PL. Bronchoscopic transparenchymal nodule access (BTPNA): first in human trial of a novel procedure for sampling solitary pulmonary nodules. Thorax 2015;70:326-32.

33. Gompelmann D. Upcoming techniques. In: Herth FJF, Shah PL, Gompelmann D, editors. Interventional Pulmonology (ERS Monograph). Sheffield, European Respiratory Society; 2017. p. 325-336.

34. Anantham D, Feller-Kopman D, Shanmugham LN, Berman SM, DeCamp MM, Gangadharan SP, et al. Electromagnetic navigation bronchoscopy-guided fiducial placement for robotic stereotactic radiosurgery of lung tumors: a feasibility study. Chest 2007;132:930-5.

35. McGuire FR, Michael Kerley J, Ochran T. Radioritheraphy monitoring device implantation into peripheral lung cancers: a therapeutic utility of electromagnetic navigational bronchoscopy. J Bronchology Interv Pulmonol 2007;14:173-6.

36. Andrade RS. Electromagnetic navigation bronchoscopy-guided thoracoscopic wedge resection of small pulmonary nodules. Semin Thorac Cardiovasc Surg 2010;22:262-5.

37. Speicher JE, Bowling MR, Anciano CJ. Bronchoscopically placed dye marking for minimally invasive thoracic surgery: a surgeon's perspective. Clin Pulm Med 2017;24:239-49.

38. Becker HD, McLemore T, Harms W. Electromagnetic navigation and endobronchial ultrasound for brachytherapy of inoperable peripheral lung cancer. Chest 2008;134:S396.

39. Harms W, Krempien R, Grehn C, Hensley F, Debus J, Becker HD. Electromagnetically navigated brachytherapy as a new treatment option for peripheral pulmonary tumors. Strahlenther Onkol 2006;182:108-11.
40. Eberhardt R, Kahn N, Herth FJ. 'Heat and destroy': bronchoscopicguided therapy of peripheral lung lesions. Respiration 2010;79:265-73.

41. Fielding D, Phillips M, Robinson P, Irving L, Garske L, Hopkins P; Thoracic Society of Australia and New Zealand. Advanced interventional pulmonology procedures: training guidelines from the Thoracic Society of Australia and New Zealand. Respirology 2012;17:1176-89.

42. Eom JS, Mok JH, Kim I, Lee MK, Lee G, Park H, et al. Radial probe endobronchial ultrasound using a guide sheath for peripheral lung lesions in beginners. BMC Pulm Med 2018;18:137.

43. Seijo LM. Electromagnetic navigation bronchoscopy: clinical utility in the diagnosis of lung cancer. Lung Cancer (Auckl) 2016;7:111-8.

44. Eberhardt R, Anantham D, Herth F, Feller-Kopman D, Ernst A. Electromagnetic navigation diagnostic bronchoscopy in peripheral lung lesions. Chest 2007;131:1800-5.

45. Mehta AC, Hood KL, Schwarz Y, Solomon SB. The evolutional history of electromagnetic navigation bronchoscopy. Chest 2018;154:935-47.

46. Mullon JJ, Burkart KM, Silvestri G, Hogarth DK, Almeida F, Berkowitz D, et al. Interventional pulmonology fellowship accreditation standards: executive summary of the multi-society interventional pulmonology fellowship accreditation committee. Chest 2017;151:1114-21.

47. Raval AA, Amir L. Community hospital experience using electromagnetic navigation bronchoscopy system integrating tidal volume computed tomography mapping. Lung Cancer Manag 2016;5:9-19.

48. Lamprecht B, Porsch P, Wegleitner B, Strasser G, Kaiser B, Studnicka $\mathrm{M}$. Electromagnetic navigation bronchoscopy (ENB): increasing diagnostic yield. Respir Med 2012;106:710-5.

49. Leong S, Ju H, Marshall H, Bowman R, Yang I, Ree AM, et al. Electromagnetic navigation bronchoscopy: A descriptive analysis. J Thorac Dis 2012;4:173-85

50. Corbetta L, Patelli M. Executive Summary of Training and Competence Standards for the Interventional Pulmonology Master Program in Italy. J Bronchology Interv Pulmonol 2018;25:6-8.

Conflicts of interest.-The authors certify that there is no conflict of interest with any financial organization regarding the material discussed in the manuscript. Article first published online: October 31, 2018. - Manuscript accepted: October 16, 2018. - Manuscript received: October 15, 2018. 